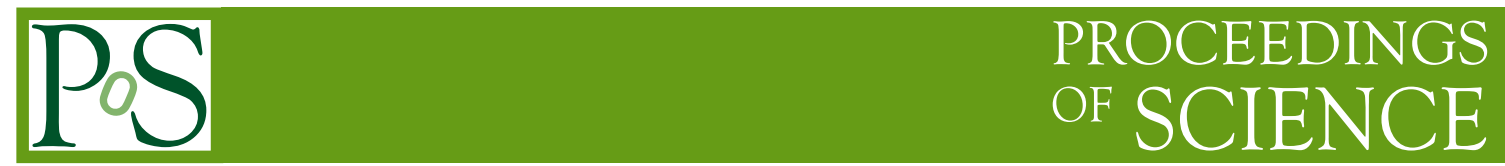

\title{
Spectroscopy of mesons with bottom quarks
}

\section{Sinéad M. Ryan*}

School of Mathematics and Hamilton Mathematics Institute, Trinity College, Dublin 2, Ireland

E-mail: ryan@maths.tcd.ie

Preliminary results for the spectra of excited and exotic bottom mesons are presented. The calculation on a dynamical anisotropic ensemble exploits distillation, enabling the use of a large basis of interpolating operators including those proportional to the gluonic field strength which are relevant for hybrid states. A comparison of results with similar calculations in the light and charm sectors is discussed.

37th International Symposium on Lattice Field Theory - Lattice2019

16-22 June 2019

Wuhan, China

${ }^{*}$ Speaker. 


\section{Introduction}

The heavy quark spectrum has proved a fertile hunting ground for exotic strongly-bound states. The discoveries, beginning more than fifteen years ago, of unexpected and surprisingly narrow charmonium-like states close to and above strong decay thresholds has spurred theoretical investigations into the nature and structure of these so-called XYZ states which confront quark models and motivate model-independent calculations of QCD to fully understand the nature of the strong force responsible for binding in hadrons. The phenomenology and experimental properties of these states are described in Ref [1], while a recent summary of lattice spectroscopy and scattering in charmonium and bottomonium is in Ref. [2] and references therein. While a resolution of the puzzles in the charmonium sector still eludes us, further puzzles appear in the bottomonium sector. To date five hadronic states with $b \bar{b}$ content have been discovered which are inconsistent with a simple $q \bar{q}$ description. While three have conventional quantum numbers - $\Upsilon(10580,10860,11020)$ - a further two, $Z_{b}(10610,10650)$, appear to require four quarks, possibly in a tetraquark or molecular arrangement. The bottomonium meson spectrum remains relatively poorly mapped out by experiments although LHCb and Belle II are expected to address this [3, 4]. Meanwhile, lattice calculations provide some information but a complete picture of the spectrum (even for the $b \bar{b}$ like states) is missing. In Refs. [5, 6] we performed an extensive calculation of the excited and exotic charmonium spectrum, determining all states up to $J=4$ including a supermultiplet of hybrid states. This motivates the exploratory study of the excited and exotic bottomonium spectrum presented here.

\section{Calculation details}

The gauge action is Symanzik-improved (anisotropic) with tree-level tadpole improved coefficients and $N_{f}=2+1$. The sea and valence quarks, including the bottom quark, are simulated with an anisotropic clover action with stout-smeared [7] spatial links. Details of the tuning of the action parameters are given in Refs. [8,9] while the mass-dependent tuning of the valence anisotropy, for a simulation with charm quarks, is described in Ref. [5]. In that latter study, the relativistic anisotropic action was successfully used to determine the excited and exotic charmonium spectrum while in Ref. [10] a similar calculation yielded the excited open-charm spectrum and Ref. [6] included an analysis of the light quark mass dependence. This study includes a mass-dependent tuning of the anisotropy in the bottomonium sector and distillation [11] to facilitate the construction of large operator bases in the relevant lattice irreducible representations (irreps, $\Lambda^{P C}$ ). Using the variational procedure the energies of states in each $\Lambda^{P C}$ are determined and exploiting a spin assignment method informed by the operator overlaps the spectrum is determined up to continuum spin $J=4$, including hybrids and states with manifestly exotic quantum numbers. Further details of the operator construction and spin identification are given in Ref. [5] and earlier work by the Hadron Spectrum Collaboration. Some relevant parameters and action details are given in Table 1.

\subsection{Dispersion relations}

On the anisotropic lattice $a_{t} m_{b}<1$, however $a_{s} m_{b}>1$ which could in principle lead to large 


\begin{tabular}{ccccccc} 
Volume & $M_{\pi}(\mathrm{MeV})$ & $\xi$ & $a_{s}$ & $a_{t}^{-1}\left(m_{\Omega}\right)$ & $N_{\text {configs. }}$ & $N_{\text {vecs }}$ \\
\hline $20^{3} \times 128$ & 391 & 3.5 & $\sim 0.12 \mathrm{fm}$ & $\sim 5.67 \mathrm{GeV}$ & 603 & 128
\end{tabular}

Table 1: Details of the ensemble used in this study. The anisotropic lattice volume corresponds to $\left(L / a_{s}\right)^{3} \times\left(T / a_{t}\right)$ while $N_{\text {configs. }}$ and $N_{\text {vecs }}$ refer to the number of gauge field configurations and the number of distillation eigenvectors respectively. This ensemble has been used in previous work by the Hadron Spectrum Collaboration, including Refs. [16, 17], where further details can be found.

discretisation errors. The effect of such artefacts is investigated by determining the dispersion relations of heavy-heavy (bottomonium) and heavy-light (B meson) systems, from which parameters including the measured anisotropy and the meson masses (rest and kinetic) are determined.

The relativistic dispersion relation of a meson on an anisotropic lattice with quantised momenta is

$$
\left(a_{t} E\right)^{2}=\left(a_{t} M\right)^{2}+\left(\frac{1}{\xi}\right)^{2}\left(a_{s} p\right)^{2}
$$

where $a_{s}$ and $a_{t}$ are the spatial and temporal lattice spacings respectively and momenta are quantised on a lattice of length $L$ with periodic boundary conditions according to $a_{s} \vec{p}=\frac{2 \pi}{L}\left(n_{x}, n_{y}, n_{z}\right)$, with $n_{i} \in\left\{0,1, \ldots, L / a_{s}-1\right\}$. In this study the heavy quark mass and the valence (heavy) anisotropy are tuned so that the dispersion relation of the $\eta_{b}$ meson, including momenta up to $(2,0,0)$, reproduces the experimentally measured $\eta_{b}$ mass and the target anisotropy $\xi=3.5$. This is shown in Figure 1, which also shows the fit parameters determined when momenta up to $(2,1,1)$ are subsequently included in a separate fit and when a term proportional to $p^{4}$ is allowed in the fits. The effect of these additional points and terms is neglible and fits yield consistent relativistic dispersion relations. Taking the bare heavy quark mass and the tuned anisotropy from the $\eta_{b}$ dispersion rela-

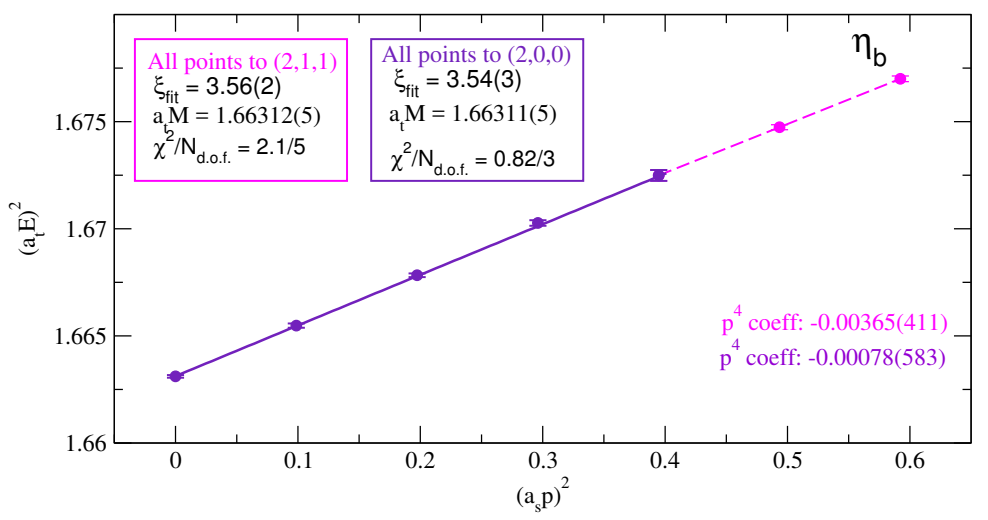

Figure 1: The dispersion relation for the $\eta_{b}$ with the input heavy quark mass tuned so that $m_{\eta_{b}}$ determined from the intercept takes its experimental value and the input anisotropy yields a measured value close to the target value of 3.5. The tuned values were taken from fits to points up to $(2,0,0)$ units of momenta (purple) although little change was seen when higher momenta (magenta) were included, as indicated. The errors are statistical only.

tion the $\Upsilon$ and the heavy-light pseudoscalar and vector dispersion relations are determined, shown in Figure 2. The measured anisotropies are consistent and in agreement with the target value, re- 
sulting in consistent rest and kinetic meson masses. Once again, higher-order terms in the lattice dispersion relation, $\mathscr{O}\left(p^{4}\right)$, were included in the fits and the effects were found to be negligible.
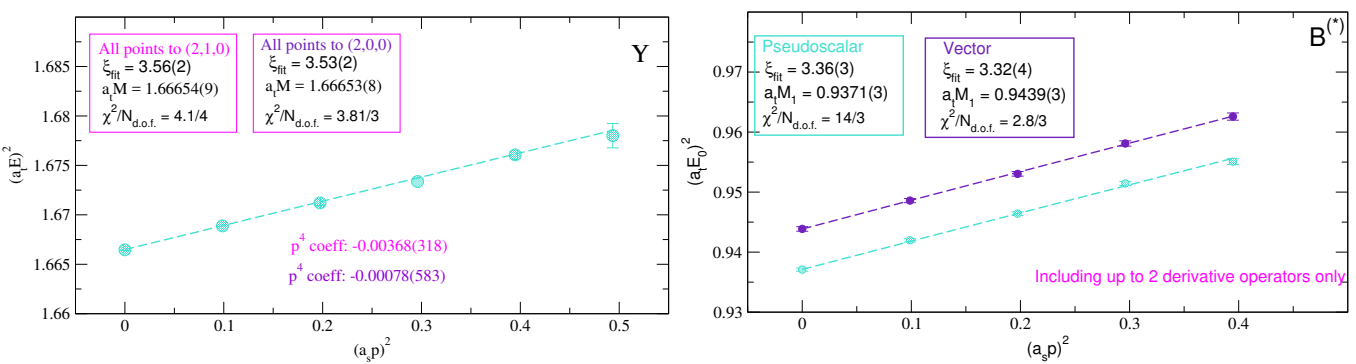

Figure 2: Dispersion relations for the $\Upsilon$ (left pane) and the heavy-light pseudoscalar and vector mesons (right pane). The bare heavy quark mass and the valence anisotropy have been determined from the $\eta_{b}$ dispersion relation. The data show no deviation from linear (relativistic) behaviour and the rest and kinetic masses determined in each case are in agreement. The anisotropy measured from the slope of the dispersion relations (shown in the boxes in each plot) is also in good agreement with the result for the $\eta_{b}$ meson.

\section{Results}

Local and non-local operators are constructed using distillation. Following Refs. [12, 13] continuum operators are constructed with definite $J^{P C}$ and lattice versions using discrete representations of the gauge-covariant derivatives are then subduced to cubic group irreps, $\Lambda^{P C}$. The distillation method facilitates the efficient calculation of large operator bases, including up to three derivatives as used in this study, from which energies and overlaps are extracted by the solution of a generalised eigenvalue problem at a suitable reference timeslice $t_{0}$. The energies are determined from fits to the time-dependence of the eigenvalues (principal correlators) according to

$$
\lambda_{n}(t)=\left(1-A_{n}\right) e^{-m_{n}\left(t-t_{0}\right)}+A_{n} e^{-m_{n}^{\prime}\left(t-t_{0}\right)},
$$

with free parameters $A_{n}, m_{n}, m_{n}^{\prime}$. An example of the principal correlators in the $T_{1}^{--}$irrep from which energy levels are determined is shown in Figure 3. The bottomonium spectrum, organised by lattice irrep is shown in Figure 4. The mass of the $\eta_{b}$ is subtracted to minimise uncertainty from the heavy quark tuning and the energy levels are colour-coded according to the spin of the continuum operator which dominates. The pattern of states is broadly similar to that determined in an earlier study of the charmonium spectrum [5] including states with the exotic quantum numbers $\left(0^{+-}, 1^{-+}, 2^{+-}\right)$found in $A_{1}^{+-}, T_{1}^{-+}$and $\left(T_{2}^{+-}, E^{+-}\right)$, and hybrid states which may have exotic or non-exotic quantum numbers. The $B_{c}$ spectrum is also determined yielding a ground state mass, $m_{B_{c}}^{\text {lattice }}=6276 \pm 1 \mathrm{MeV}$, which can be compared with the experimental value [14], 6274.9 \pm 0.8 $\mathrm{MeV}$. A more extensive calculation of the $B_{c}$ spectrum is underway.

\subsection{The hybrid mesons in bottomonium}

Following earlier studies in the light, open-charm and charmonium systems operators with non-trivial gluonic content are included in the bases. By analysing the operator overlaps candidate 

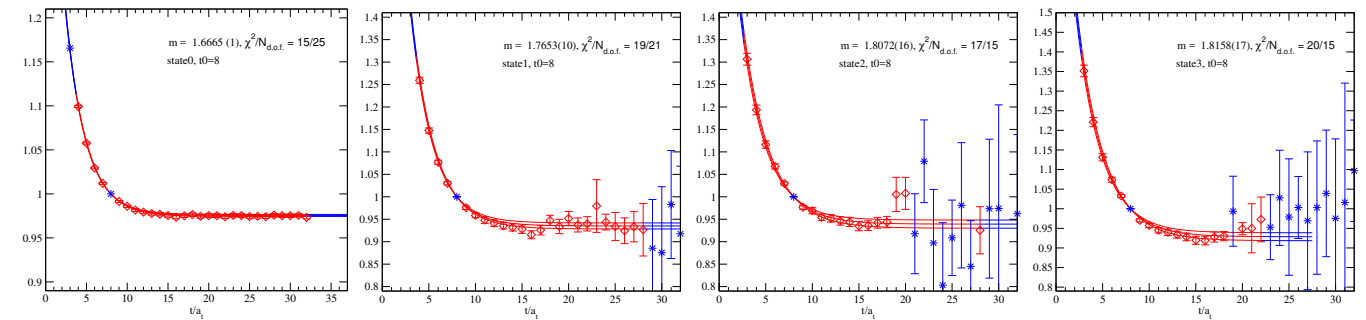

Figure 3: The principal correlator fits, for the ground, first, second and third excited states in the $T_{1}^{--}$irrep in this work. The data points are $\lambda^{n}(t) \cdot e^{m_{n}\left(t-t_{0}\right)}$ with a reference timeslice $t_{0}=8$ as determined from fits to Eqn 3.1. The extracted mass in lattice units together with the $\chi^{2} / N_{\text {d.o.f. }}$ are shown for each fit. The leading time dependence due to state $n$ has been divided out in each case, which results in a straight line at unity when a single exponential dominates the fit.

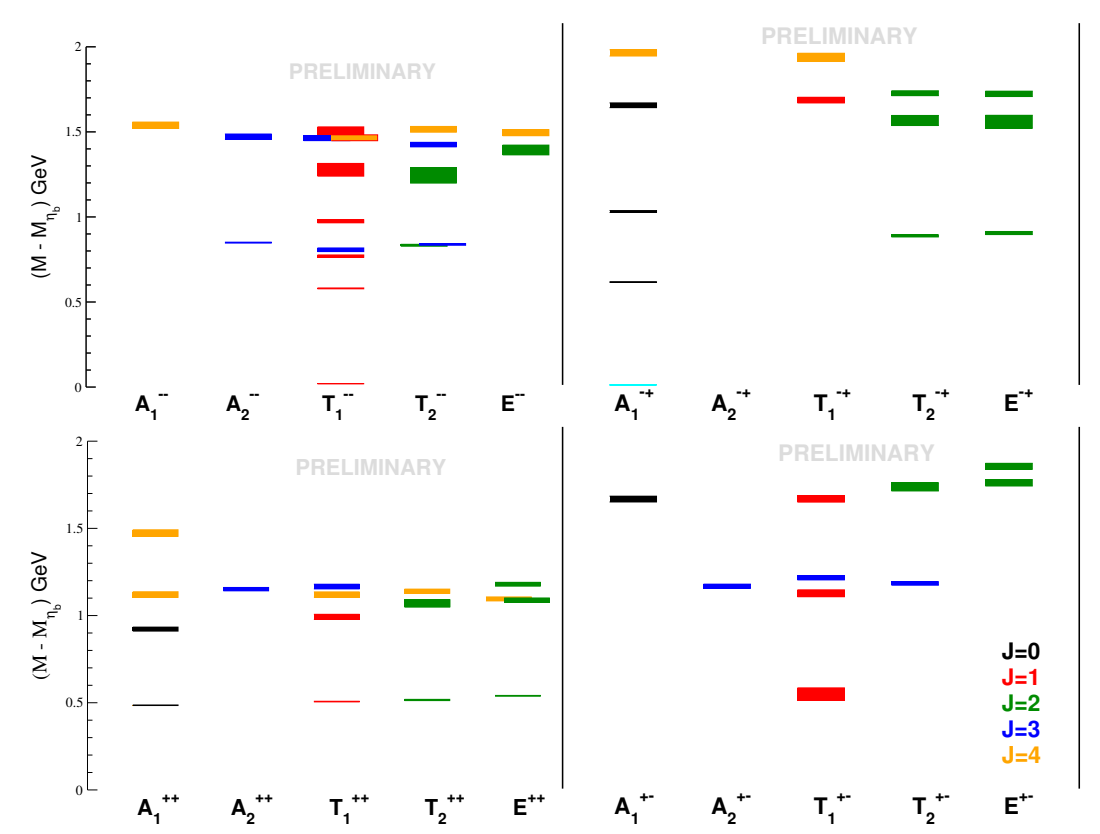

Figure 4: The bottomonium spectrum, labelled by lattice irrep $\Lambda^{P C}$, determined in this study. The vertical height of the boxes represents the one-sigma statistical uncertainty about the mean determined from fits of principal correlators to Eqn 3.1. Colours indicate the continuum spin. The spectrum is presented as mass splittings with the $\eta_{b}$ meson to reduce the uncertainty from tuning the heavy quark mass.

hybrid mesons are identified when the state is dominated by a hybrid-like operator. In particular, three states are found which do not seem to fit the pattern expected from quark models with nonexotic quantum numbers, in the $T_{1}^{--}, A_{1}^{-+}$and $\left(T_{2}, E\right)^{-+}$irreps. These "excess" states have a significantly larger overlap with operators proportional to the gluonic field strength tensor, $F^{\mu v}$ than the states in the same irreps which conform to a quark model picture. An exotic hybrid meson is also identified in the the $T_{1}^{-+}$irrep which is again dominated by operators proportional to $F^{\mu v}$. The energy levels in these lattice irreps are shown in Figure 5 with the hybrid candidates shown in red. The energy scale for the bottomonium hybrids (the splitting of the lowest-lying hybrid with the 


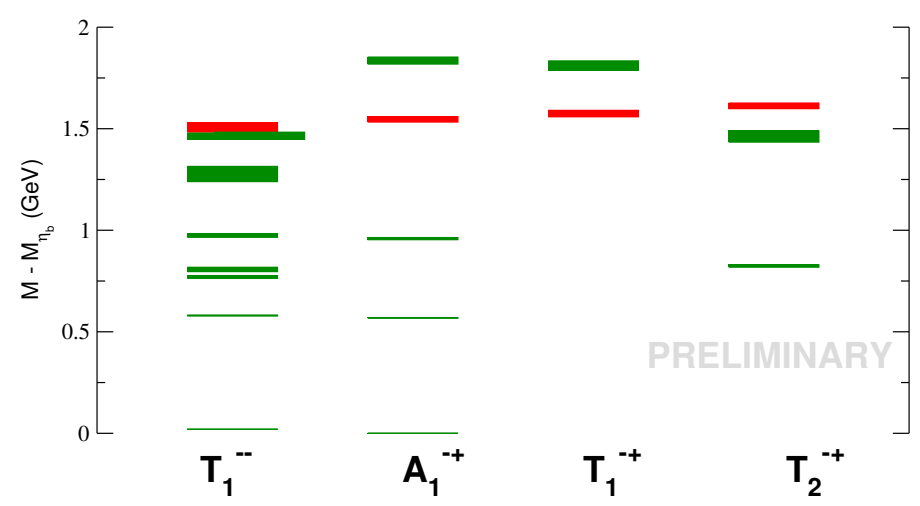

Figure 5: The lattice irreps in which candidate hybrid mesons are identified, by examining the operator-state overlaps. The energy levels are the same as those shown in Figure 4 with the hybrids shown in red.

lightest state in the system) is approximately $1.5 \mathrm{GeV}$, similar to that found in the light, heavy-light and charmonium meson and baryon sectors and suggesting similar dynamics at play. Although still preliminary and not presented at this conference, the same pattern of hybrids is also found in a analysis of the B-meson system, on the same ensemble. These results also agree well with a recent determination of the spin-structure of bottomonium hybrids [15] using non-relativistic effective field theory and input from the lattice determination of the charmonium hybrids [5].

Looking in more detail at the operator overlaps in the lattice irreps in which hybrids have been identified and which were subduced from hybrid-like operators of the form $\left\{\gamma_{5}, \gamma_{i}\right\} \times D_{J=1}^{[2]}$, a common absolute value of the overlap $|Z|$ is found, encouraging the identification of a lightest hybrid supermultiplet of bottomonium hybrids - corresponding to those shown in Figure 5 - with $J^{P C}=(0,1,2)^{-+}, 1^{--}$. The overlaps determined in bottomonium channels are shown in Figure 6 and agree very well with the similar study of charmonium, already mentioned [5].

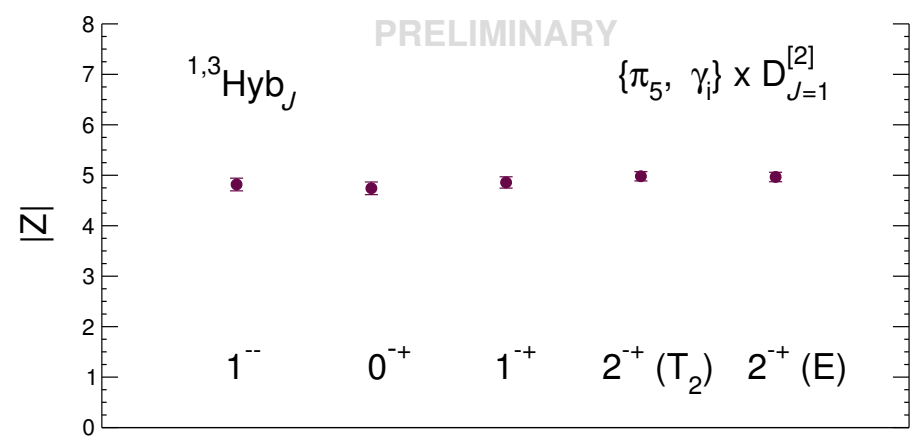

Figure 6: The operator overlaps, $|Z|$, for the proposed lightest hybrid supermultiplet in bottomonium.

\section{Summary}

An exploratory determination of the bottomonium spectrum is presented, including excited and exotic states up to $J=4$. The calculation is performed on the Hadron Spectrum Collabora- 
tion's $N_{f}=2+1$ dynamical anisotropic ensembles and fermions, including the bottom quark, are simulated with the same relativistic action. The dispersion relations of pseudoscalar and vector heavy-heavy and heavy-light mesons show no evidence of significant discretisation errors. An extensive spectrum of states is determined and includes the identification of a hybrid supermultiplet at an energy scale similar to that found for hybrids in the light, heavy-light and charmonium sectors. Further work is ongoing, including a determination of the $B$ and $B_{c}$ meson spectra.

\section{Acknowledgements}

SMR thanks colleagues in the Hadron Spectrum Collaboration and is grateful to DAMTP, the University of Cambridge and the Technische Universität München for hospitality while this work was ongoing.

\section{References}

[1] R. F. Lebed, R. E. Mitchell and E. S. Swanson, Prog. Part. Nucl. Phys. 93 (2017) 143 [arXiv:1610.04528 [hep-ph]].

[2] M. Padmanath, PoS LATTICE 2018 (2018) 013 [arXiv:1905.09651 [hep-lat]].

[3] E. Kou et al. [Belle-II Collaboration], arXiv:1808.10567 [hep-ex].

[4] R. Aaij et al. [LHCb Collaboration], arXiv:1808.08865.

[5] L. Liu et al. [Hadron Spectrum Collaboration], JHEP 1207 (2012) 126 [arXiv:1204.5425 [hep-ph]].

[6] G. K. C. Cheung et al. [Hadron Spectrum Collaboration], JHEP 1612 (2016) 089 [arXiv:1610.01073 [hep-lat]].

[7] C. Morningstar and M. J. Peardon, Phys. Rev. D 69 (2004) 054501 [hep-lat/0311018].

[8] R. G. Edwards, B. Joo and H. W. Lin, Phys. Rev. D 78 (2008) 054501 [arXiv:0803.3960 [hep-lat]].

[9] H. W. Lin et al. [Hadron Spectrum Collaboration], Phys. Rev. D 79 (2009) 034502 [arXiv:0810.3588 [hep-lat]].

[10] G. Moir, M. Peardon, S. M. Ryan, C. E. Thomas and L. Liu, JHEP 1305 (2013) 021 [arXiv:1301.7670 [hep-ph]].

[11] M. Peardon et al. [Hadron Spectrum Collaboration], Phys. Rev. D 80 (2009) 054506 [arXiv:0905.2160 [hep-lat]].

[12] J. J. Dudek, R. G. Edwards, M. J. Peardon, D. G. Richards and C. E. Thomas, Phys. Rev. Lett. 103 (2009) 262001 [arXiv:0909.0200 [hep-ph]].

[13] J. J. Dudek, R. G. Edwards, M. J. Peardon, D. G. Richards and C. E. Thomas, Phys. Rev. D 82 (2010) 034508 [arXiv:1004.4930 [hep-ph]].

[14] M. Tanabashi et al (Particle Data Group), Phys. Rev. D 98 (2018) 030001.

[15] N. Brambilla, W. K. Lai, J. Segovia, J. Tarrús Castellà and A. Vairo, Phys. Rev. D 99 (2019) no.1, 014017 [arXiv: 1805.07713 [hep-ph]].

[16] C. O’Hara, S. M. Ryan, G. Moir and C. E. Thomas, PoS Lattice 2016 (2016) 120 [arXiv:1702.00352 [hep-lat]].

[17] J. J. Dudek et al. [Hadron Spectrum Collaboration], Phys. Rev. D 88 (2013) no.9, 094505 [arXiv:1309.2608 [hep-lat]]. 